\title{
Trichotillomania Masked by Diffuse Alopecia Areata: A Case Report
}

\author{
Val Constantine S. Cua, MD, ${ }^{1}$ Felix Paolo J. Lizarondo, $\mathrm{MD}^{1}$ and Claudine Y. Silva, MD² \\ ${ }^{1}$ Department of Dermatology, Philippine General Hospital, University of the Philippines Manila \\ ${ }^{2}$ Department of Dermatology, College of Medicine and Philippine General Hospital, University of the Philippines Manila
}

\begin{abstract}
An 11-year-old girl previously treated for tinea capitis presented a 3-month history of continuous decrease in hair density on the vertex, frontal, and parieto-temporal areas of the scalp. Hair pull test was negative. Trichoscopic findings showed black dots, micro-exclamation point hairs, regrowing vellus hair, and zigzag hairs. Histopathology showed $\mathrm{CD}^{+}$peribulbar lymphocytic infiltrates and occasional eosinophils around the anagen hair follicle consistent with a non-scarring alopecia. A diagnosis of diffuse alopecia areata was made. Patient was given methylprednisolone $(0.5 \mathrm{mg} / \mathrm{kg} / \mathrm{day})$ for 2 weeks and noted marked increase in hair density except on focal areas of the scalp. Patient eventually admitted to occasional hair pulling. Trichoscopy revealed trichoptilosis, V-sign, tulip hairs, and multiple broken hairs of varying length while a second biopsy showed trichomalacia and pigment casts consistent with trichotillomania. In this case, where co-existence of alopecia areata and trichotillomania is considered to be uncommon, trichoscopy proved to be an important tool in differentiating hair disorders with similar presentation. Knowing key features of hair diseases can help elucidate the diagnosis when presented with an atypical case.
\end{abstract}

Key Words: alopecia areata, trichotillomania, trichoscopy

\section{INTRODUCTION}

Poster presented in the $2^{\text {nd }}$ Annual Meeting of Asian Society of Dermatopathology on November 13-15, 2020, at Kaohsiung, Taiwan.

Poster presented in the $29^{\text {th }}$ European Academy of Dermatology and Venereology Virtual Congress on October 29-31, 2020.

Poster presented in the $42^{\text {nd }}$ Philippine Dermatological Society Annual Convention and $10^{\text {th }}$ Asian Society for Pigment Cell Research Congress on November 5-7, 2019, Mandaluyong, Philippines.

Poster presented (Oral Presentation) in the $120^{\text {th }}$ Annual Meeting of the Japanese Dermatological Association on June 10-13, 2021, at Pacifico, Yokohama, Japan.

Poster presented (Digital Presentation) in the $120^{\text {th }}$ Annual Meeting of the Japanese Dermatological Association on June 10-13, 2021, at Pacifico, Yokohama, Japan.

Corresponding author: Val Constantine S. Cua, MD

Department of Dermatology

Philippine General Hospital

University of the Philippines Manila

Taft Avenue, Ermita, Manila 1000, Philippines

Email:vscua@up.edu.ph
Alopecia in pediatric patients is due to a range of congenital and acquired conditions that can either be cicatricial or non-cicatricial. ${ }^{1}$ Etiology may range from disorders of the hair shaft or hair follicle, to infectious and autoimmune diseases. A thorough history provides information regarding acuity and pattern of hair loss, associated comorbidities, and nutritional status. Physical examination may reveal signs of inflammation and the extent of hair-bearing regions involved, while maneuvers such as the tug test and hair pull test provide a measure of hair fragility and disease activity, respectively. Trichoscopy allows for a non-invasive analysis of several follicular patterns as well as hair signs and shaft features that can help narrow down differential diagnoses among hair disorders that present similarly. ${ }^{2}$ Furthermore, it can be utilized to monitor disease activity and therapeutic response. The utility of trichoscopy is highlighted in this case where co-existence of alopecia areata and trichotillomania was uncovered after correlating clinical and histologic findings with the trichoscopic patterns.

\section{CASE DESCRIPTION}

An 11-year-old girl previously treated for atopic dermatitis and tinea capitis presented with a 3-month history of continuous decrease in hair density on the vertex and fronto-parieto-temporal areas of the scalp (Figure 1A, 


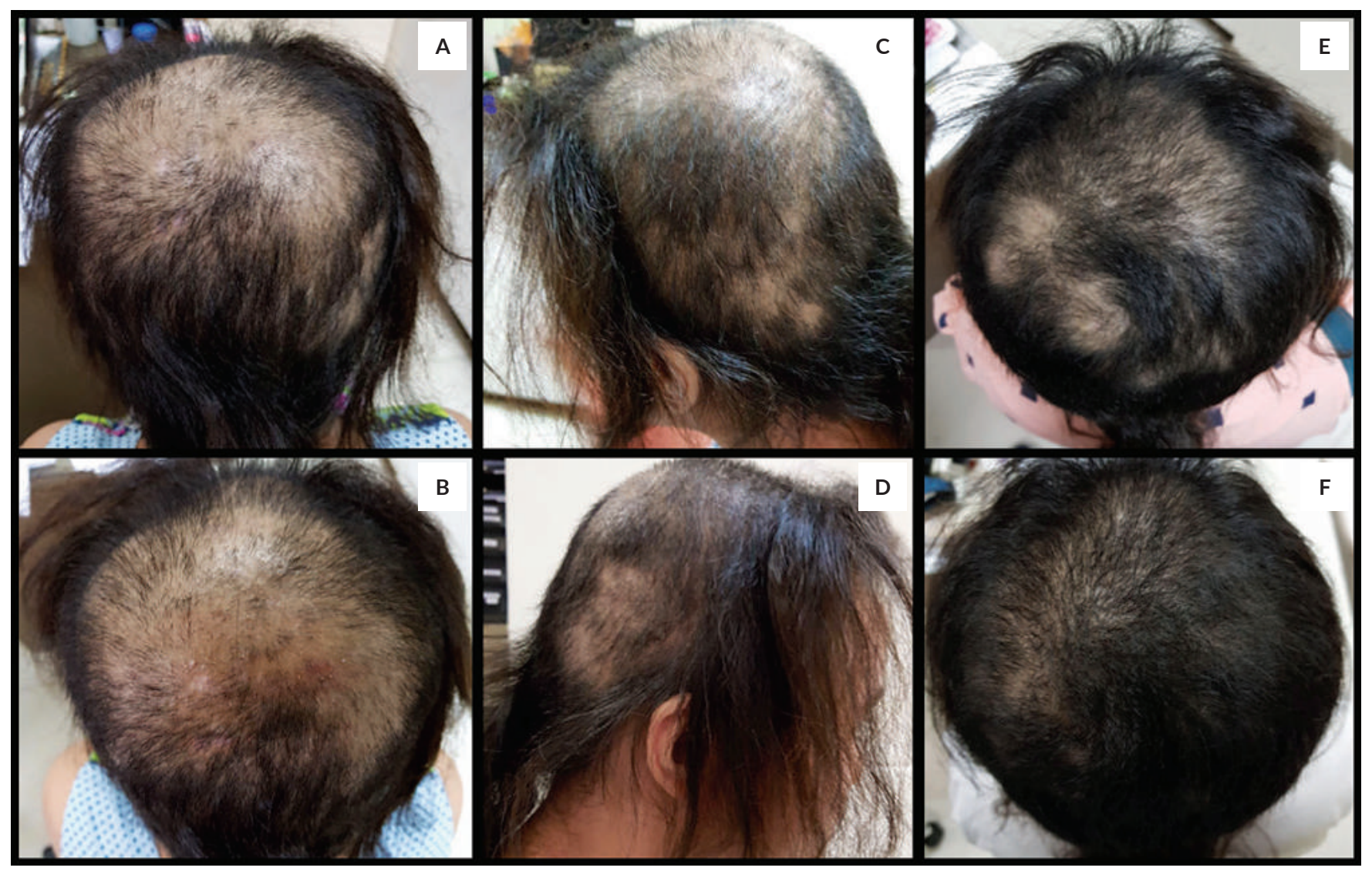

Figure 1. (A) Posterior view of the scalp showed diffuse alopecic patch with varying lengths of broken hairs on the vertex. (B) Top view emphasized the varying length of broken hair up to the frontal area and some erythematous papules. (C, D) Decrease in hair density was also observed with focal areas of alopecia on the temporo-parietal area of the scalp. (E) After 1 month of methylprednisolone $(0.5 \mathrm{mg} / \mathrm{kg} /$ day), there was marked increase in hair density. However, there were still focal alopecic areas for which the patient admitted to hair pulling. (F) Continued intake of methylprednisolone for another month and hair pulling restriction resulted to almost complete regrowth of hair on the scalp.

B, C, D) associated with occasional pruritus. Hair pull test was negative. Trichoscopic findings showed black dots, micro-exclamation point hairs, regrowing vellus hair, and zigzag hairs (Figure 2A, B, C). Thyroid function tests and hematologic work-up were unremarkable. The initial biopsy section (Figure 3A, B, C, D) showed peribulbar $\mathrm{CD}^{+}$ lymphocytic infiltrates and occasional eosinophils around the anagen hair follicle. A diagnosis of diffuse alopecia areata was made. Patient was initially given a 2 -week course of oral methylprednisolone $(0.5 \mathrm{mg} / \mathrm{kg} /$ day $)$ and noted marked increase in hair density except on focal areas of the scalp. Patient eventually admitted to occasional hair pulling. Repeat trichoscopy revealed trichoptilosis, V-sign, tulip hairs, and multiple broken hairs of varying length (Figure 2D, E, D). A second biopsy on the focal alopecic area of the scalp showed trichomalacia and pigment casts (Figure 3E, F) consistent with trichotillomania. Patient was then referred to psychiatry for behavioral management. While tapering her methylprednisolone dose, she was lost to follow-up.

\section{DISCUSSION}

Diffuse alopecia areata is a special variant of alopecia areata (AA) that presents with acute onset of widespread hair rarefaction as compared to the focal alopecic patches of the typical AA. ${ }^{3}$ Current studies regarding pathophysiology involve an autoimmune process that targets the anagen stage hair follicles, thereby causing dystrophic hair growth. ${ }^{4}$ All age and ethnicities are equally affected, but a female preponderance was noted for diffuse AA. ${ }^{5}$ Based on the data available from the Philippine Dermatological Society-Health Information Systems (PDS-HIS), there were only 10 cases of diffuse AA noted from 2011-2018, with more women affected than men. At present, the cumulative lifetime incidence of $\mathrm{AA}$ is $2.1 \% .{ }^{6}$ Autoimmune diseases such as atopy, vitiligo, and diabetes increase the risk of developing AA.,7

Clinically, diffuse AA presents with a brief course of rapid generalized thinning of the parietal and anteriortemporal regions, and a positive hair pull test. ${ }^{5,8}$ The hallmark trichoscopic features of AA are regularly distributed micro- 




Figure 2. (A) Trichoscopy showed multiple broken hairs with different lengths. Increased fragility of the hair strands was demonstrated by the zigzag hair (purple circle). Other findings include (B) two micro-exclamation mark hairs (light blue circles), a regrowing vellus hair (dark blue circle), (C) multiple black dots (pink circles), (D) trichoptilosis on short hair strands (green circles), (E) V-sign formed when two hair shafts emerging from one follicular unit were pulled and broken at the same length above the scalp surface (red circle), and (F) tulip hair formed from a diagonally fractured hair (orange circle).

exclamation mark hairs, yellow dots, black dots (or cadaverous hairs), broken hairs, and regrowing vellus hairs. ${ }^{9}$ In contrast, diffuse AA do not present with all of these characteristics features. Alessandrini et al. ${ }^{8}$ concluded that among Italian patients with diffuse AA, the most frequent trichoscopic patterns were black dots, dystrophic hairs, and empty yellow dots. Recently, other subtypes of diffuse AA have been identified albeit without consensus criteria. Rebora ${ }^{10}$ coined the term alopecia areata incognito (AAI), which presents like telogen effluvium with a positive pull test and scalp dysesthesia (trichodynia), but absent hair rarefaction. Alessandrini and colleagues $^{8}$ further differentiated this from diffuse AA with the common location being at the occipital and parietal regions, and with the trichoscopic features of empty yellow dots, yellow dots with vellus hair, small regrowing hair, and pigtail hair. Another subtype is called acute diffuse and total alopecia (ADTA), which presents as diffuse hair loss and then total alopecia of the scalp within 2 days. ${ }^{11}$ Most patients are female who may complain of scalp pruritus or burning sensation prior to disease manifestation. Allergy and tissue eosinophilia are prominent features. Like AAI, nail changes and micro-exclamation mark hairs are uncommon. As with all types of diffuse AA, ADTA heralds a good prognosis and can spontaneously resolve without treatment. ${ }^{12}$
Trichotillomania (TTM) is a condition manifested as repetitive hair pulling from the scalp and other hair-bearing areas. ${ }^{13}$ The peak age is from 9 to 13 years and affecting mostly girls. The latter data is consistent with PDS-HIS data which showed male-female ratio of 1:4.3. From 2011-2018, a total of 146 cases have been recorded. Patients with TTM usually present with focal non-scarring alopecic patches with uneven borders, minimal to absent secondary lesions, varying lengths of broken strands, and a negative hair pull test. The occiput is frequently spared due to the pain of hair pulling on this area. The typical trichoscopic features include irregularly broken hairs, trichoptilosis on short hairs, contracted and coiled hairs, hair powder or residue, black dots, flame hairs, V-sign, and tulip hairs. ${ }^{9,14}$ Black dots may also be seen similar to that of AA. Although micro-exclamation mark hairs are uncommon in TTM, its presence is distinguished from that of AA by the blunt distal ends in contrast to the irregular frayed distal ends of the latter. ${ }^{9}$

Co-occurrence of AA and TTM is uncommon. ${ }^{15-18}$ Focal AA may present similarly with TTM and should be distinguished based on the trichoscopy findings mentioned earlier. In our case, TTM was not initially considered due to the diffuse hair rarefaction of the patient and the presence of micro-exclamation mark hairs and black dots. 


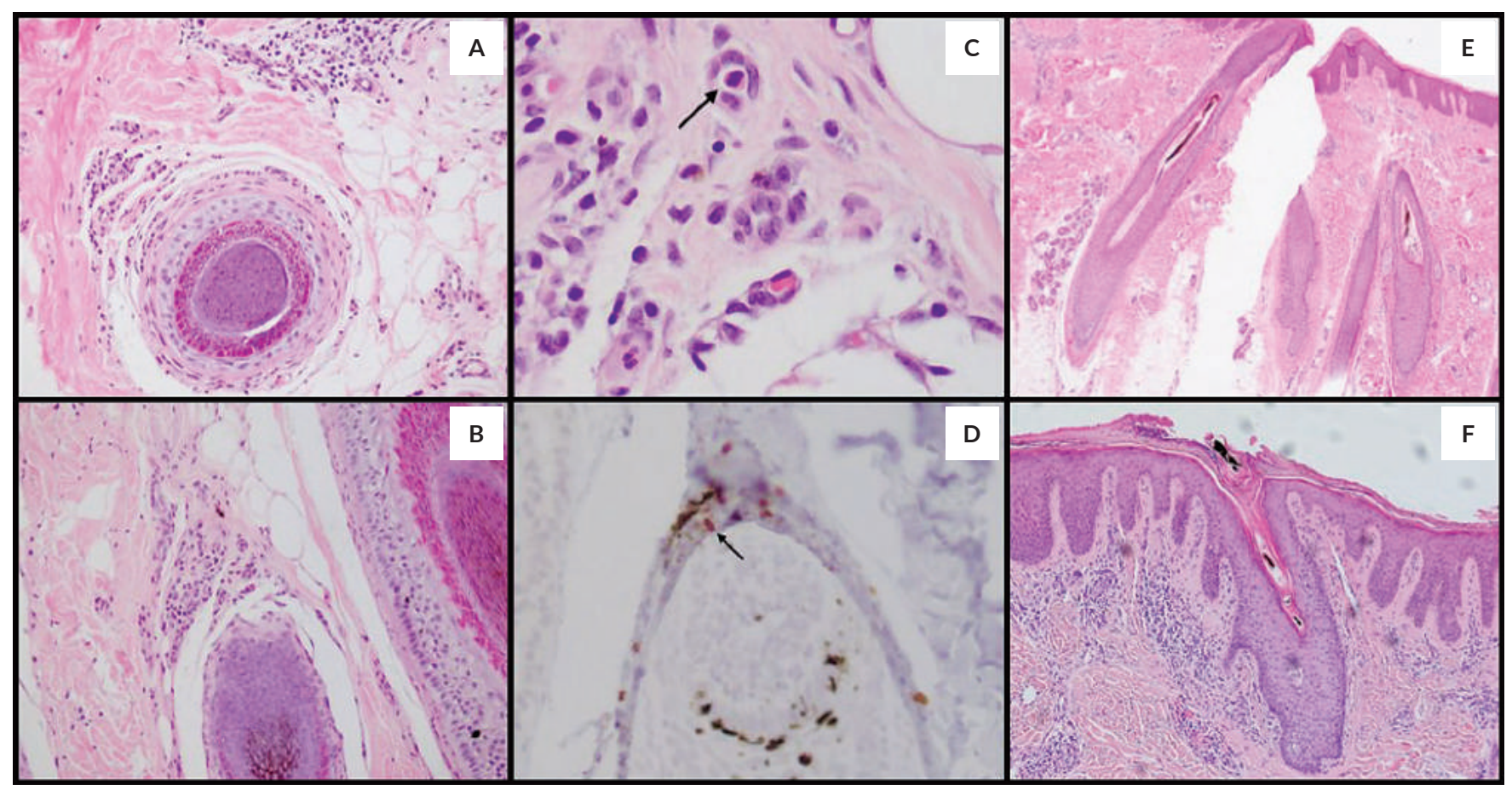

Figure 3. Histopathology (with H\&E stain) of the alopecic patch on the scalp showed peribulbar lymphocytic infiltrates surrounding the anagen hair follicle in the horizontal (A) and vertical (B) section (20X) consistent with non-scarring alopecia. (C) A closer view (40X) of the section in (B) shows occasional eosinophils (arrow) surrounding the hair bulb. (D) CD3+ T-lymphocytes were stained (40X) in the vertical section of (B), which is commonly seen in alopecia areata. A second biopsy (10X) from the remaining alopecic patch on the scalp showed trichomalacia $(E)$ and pigment casts (F) consistent with trichotillomania.

Reviewing the trichoscopic findings, however, did point to early signs of TTM such as broken hairs of different lengths, trichoptilosis on short hairs, V-sign, and tulip hairs. This diagnosis was also further supported by biopsy findings of trichomalacia and pigment casts. A negative hair pull test was inconsistent with diffuse AA in literature but marked hair growth response after a month of methylprednisolone verified the autoimmune etiology of the hair disease. The negative hair pull test may also be explained in part by few peribulbar lymphocytic and occasional eosinophilic infiltrates, indicating that the disease may be in the subacute stage or recovery stage. ${ }^{19,20}$ Lastly, a positive CD3 immunostaining of peribulbar lymphocytes distinguished our patient's case from that of non-autoimmune hair diseases such as telogen effluvium and female pattern baldness. ${ }^{20}$

\section{CONCLUSION}

Co-existence of alopecia areata and trichotillomania is uncommon. Trichoscopy and pathological features are important diagnostics in differentiating hair disorders with similar presentation. Knowing key features of hair diseases can help elucidate the diagnosis when presented with an atypical case.

\section{Statement of Authorship}

All authors participated in the data collection and analysis, and approved the final version submitted.

\section{Author Declaration}

All authors declared no conflicts of interest.

\section{Funding Source}

This paper was self-funded.

\section{REFERENCES}

1. Xu L, Liu KX, Senna MM. A practical approach to the diagnosis and management of hair loss in children and adolescents. Front Med. 2017; 4:112.

2. Rudnicka L, Rakowska A, Olszewska M. Trichoscopy. How it may help the clinician. Dermatol Clin. 2013; 31(1):29-41.

3. Zhao Y, Zhang B, Caulloo S, Chen X, Li Y, Zhang X. Diffuse alopecia areata is associated with intense inflammatory infiltration and $\mathrm{CD} 8+\mathrm{T}$ cells in hair loss regions and an increase in serum IgE level. Indian J Dermatol Venereol Leprol. 2012; 78(6):709-14.

4. Kang H, Wu W-Y, Lo BKK, Mei Y, Leung G, Shapiro J, et al. Hair follicles from alopecia areata patients exhibit alterations in immune privilege-associated gene expression in advance of hair loss. J Invest Dermatol. 2010; 130(11):2677-80.

5. Khan Mohammad Beigi P. Diffuse Alopecia Areata. In: Alopecia Areata: A Clinician's Guide. Cham: Springer International Publishing; 2018:9-11. 
6. Mirzoyev SA, Schrum AG, Davis MDP, Torgerson RR. Lifetime incidence risk of alopecia areata estimated at $2.1 \%$ by Rochester Epidemiology Project, 1990-2009. J Invest Dermatol. 2014; 134(4):1141-2.

7. Lee $\mathrm{S}$, Lee H, Lee CH, Lee W-S. Comorbidities in alopecia areata: a systematic review and meta-analysis. J Am Acad Dermatol. 2019; 80(2):466-77.e16.

8. Alessandrini A, Starace M, Brandi N, Bruni F, Piraccini B. Trichoscopic findings in alopecia areata incognita and diffuse alopecia areata: a 5-year Italian study. In: 24th World Congress of Dermatology. Bologna; 2019.

9. Rudnicka L, Olszewska M, Rakowska A. Atlas of Trichoscopy: Dermoscopy in Hair and Scalp Disease. London: Springer; 2012.

10. Rebora A. Alopecia areata incognita: a hypothesis. Dermatology. 1987; 174(5):214-8.

11. Sato-Kawamura M, Aiba S, Tagami H. Acute diffuse and total alopecia of the female scalp: a new subtype of diffuse alopecia areata that has a favorable prognosis. Clin Lab Investig. 2002; 205:367-73.

12. Lew BL, Shin MK, Sim WY. Acute diffuse and total alopecia: A new subtype of alopecia areata with a favorable prognosis. J Am Acad Dermatol. 2009; 60(1):85-93.
13. American Psychiatric Association. Diagnostic and Statistical Manual of Mental Disorders. 5th ed. Washington, D.C.: American Psychiatric Association; 2013.

14. Rakowska A, Slowinska M, Olszewska M, Rudnicka L. New trichoscopy findings in trichotillomania: flame hairs, v-sign, hook hairs, hair powder, tulip hairs. Acta Derm Venereol. 2014; 94(3):303-6.

15. Bhalla M, Sarkar R, Arun P, Kanwar AJ. Trichotillomania. Indian Pediatr. 2003; 40(1):52-6.

16. Trüeb RM, Cavegn B. Trichotillomania in connection with alopecia areata. Cutis. 1996; 58(1):67-70.

17. Wilkin JK. Trichotillomania associated with alopecia areata. Cutis. 1983; 31(1):65-6.

18. Brzezinski $\mathrm{P}$, Cywinska $\mathrm{E}$, Chiriac A. report of a rare case of alopecia areata coexisting with trichotillomania. Int J Trichology. 2016; 8(1): $32-4$.

19. Whiting DA. Histopathologic features of alopecia areata. Arch Dermatol. 2003; 139(12):1555-9.

20. Kolivras A, Thompson C. Distinguishing diffuse alopecia areata (AA) from pattern hair loss (PHL) using CD3+ T cells. J Am Acad Dermatol. 2016; 74(5):937-44.

The Acta Medica Philippina is now accepting original scientific papers, review articles and case reports for its upcoming issues. Please follow the format for submission as indicated in the "Instructions to Authors" elsewhere in the journal. All papers received shall be properly acknowledged. For inquiries and submission of proposals, please email us at actamedicaphilippina.upm@up.edu.ph 